\title{
Structural Markers of the Frenkel Line in the Proximity of Widom Lines Supporting Information
}

Ciprian G. Pruteanu, ${ }^{*}{ }^{\dagger}, \|$ John E. Proctor, ${ }^{*} \neq$ Oliver L.G. Alderman, $,{ }^{*}, \pi$ and John S. Loveday*§

†Department of Earth Sciences, University College London, Gower Street, London WC1E6BT, United Kingdom

‡Materials \& Physics Research Group, Newton Building, University of Salford, Manchester M5 4WT, United Kingdom

ףISIS Neutron and Muon Source, Rutherford Appleton Laboratory, Chilton, Didcot, Oxford OX11 0QX, United Kingdom

$\S S U P A$, School of Physics and Astronomy and Centre for Science at Extreme Conditions, The University of Edinburgh, Edinburgh EH9 3JZ, United Kingdom INow at SUPA, School of Physics and Astronomy and Centre for Science at Extreme Conditions, The University of Edinburgh, Edinburgh EH9 3JZ, United Kingdom

E-mail: cip.pruteanu@ed.ac.uk; j.e.proctor@salford.ac.uk; oliver.alderman@stfc.ac.uk; j.loveday@ed.ac.uk

\section{Contents}

- Analysis of the $\mathrm{N} \equiv \mathrm{N}$ triple bond from the intramolecular neutron PDFs

- Experimental structure factors for $\mathrm{N}_{2}$ at $160 \mathrm{~K}$ - SANDALS Instrument, ISIS, RAL, UK

Analysis of the $\mathrm{N} \equiv \mathrm{N}$ triple bond from the intramolecular neutron PDFs

Total correlation functions were obtained by sine Fourier transformations of the experimental $S(Q)$ :

$T(r)=T^{0}(r)+D(r)$

Where $\mathrm{D}(\mathrm{r})=\frac{2}{\pi} \int_{0}^{Q_{\max }} Q(S(Q)-1) M(Q) \sin (r Q) \mathrm{d} Q$

$T^{0}(r) / r=4 \pi \rho_{0} b_{N}{ }^{2}, M(Q)$ is the Lorch modification function, $b_{N}=9.36(2) \mathrm{fm}$ is the coherent neutron scattering length for nitrogen and $\rho_{0}$ is the atom number density.

The $T(r)$ were fitted in the region of the $N \equiv N$ intramolecular peak over the range $0.6 \leq r \leq 1.6 \AA$ using the program PFIT. ${ }^{1}$ The $Q_{\max }$ value used determines the real-space resolution and was optimized by fitting the $125 \mathrm{MPa}$ dataset as a function of $Q_{\max }$ to yield a value of $27.86 \AA^{-1}$, which corresponds approximately to where the $\mathrm{N} \equiv \mathrm{N}$ intramolecular coherent scattering can no longer be distinguished from background noise. Both the $160 \mathrm{~K}$ data of the present study, as well as previously reported data at $300 \mathrm{~K}^{2}$ were fitted in the same way.

No clear correlations with pressure or temperature were observed in any fitting parameters, and thus we report the mean values obtained in Table S1.

Table S1: Mean parameters of the $N \equiv N$ intramolecular bond obtained by averaging over the $T(r)$ fitting results at all pressures. Standard deviations on final digit in parentheses.

\begin{tabular}{ccccc}
\hline$T(K)$ & $\begin{array}{c}\text { P range } \\
(\mathrm{MPa})\end{array}$ & $\mathrm{r}_{\mathrm{N} \equiv \mathrm{N}}(\AA)$ & $\begin{array}{c}\left\langle\mathrm{U}_{\mathrm{N} \equiv \mathrm{N}^{2}}\right\rangle^{1 / 2} \\
(\AA)\end{array}$ & $\mathrm{n}_{\mathrm{N} \equiv \mathrm{N}}$ \\
\hline 160 & $7.8-125$ & $1.098(4)$ & $0.050(7)$ & $0.86(2)$ \\
300 & $25-300$ & $1.101(7)$ & $0.048(5)$ & $0.82(5)$ \\
\hline
\end{tabular}


The lack of pressure or temperature dependence in the fitting parameters implies that any true variations are below the sensitivity of the measurements. This is consistent with the variation in bond length inferred from Raman spectroscopy, ${ }^{2}$ which over the pressure range explored at $300 \mathrm{~K}$ by neutron diffraction measurements, passes through a maximum, with a total variation of a few tenths of a picometer, on the order of the standard deviations on $\mathrm{r}_{\mathrm{N} \equiv \mathrm{N}}$ reported in Table S1. In order to observe the small changes involved by diffraction, both exploration of a much larger pressure interval, as well as greatly improved counting statistics would be required.

\section{Experimental structure factors for $\mathrm{N}_{2}$ at $160 \mathrm{~K}$ - SANDALS Instrument, ISIS, RAL, UK}

Below, we present the reduced experimental structure factors $(S(q)-1$, for easier comparison to EPSR fits) and their associated EPSR ${ }^{3}$ fits for all the runs from which the pair distribution functions and coordination numbers were obtained for the main paper. The fits are only shown out to $30 \mathrm{~A}^{-1}$ to improve readability of the low- $Q$ region (the full raw patterns can be found in the supplementary materials provided). Raw S(q) data can be found at: https://data.isis.stfc.ac.uk/doi/study/111245768 

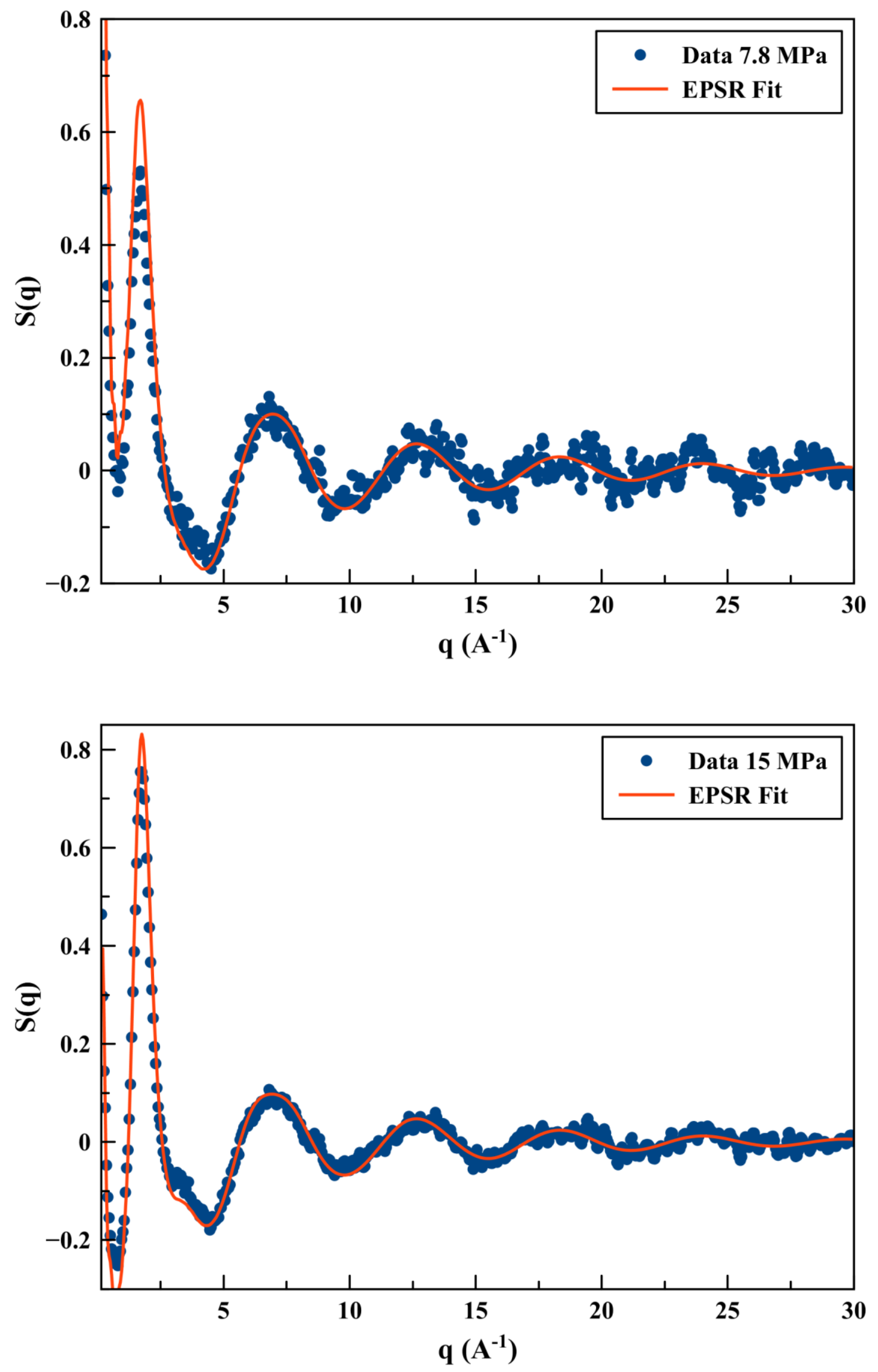

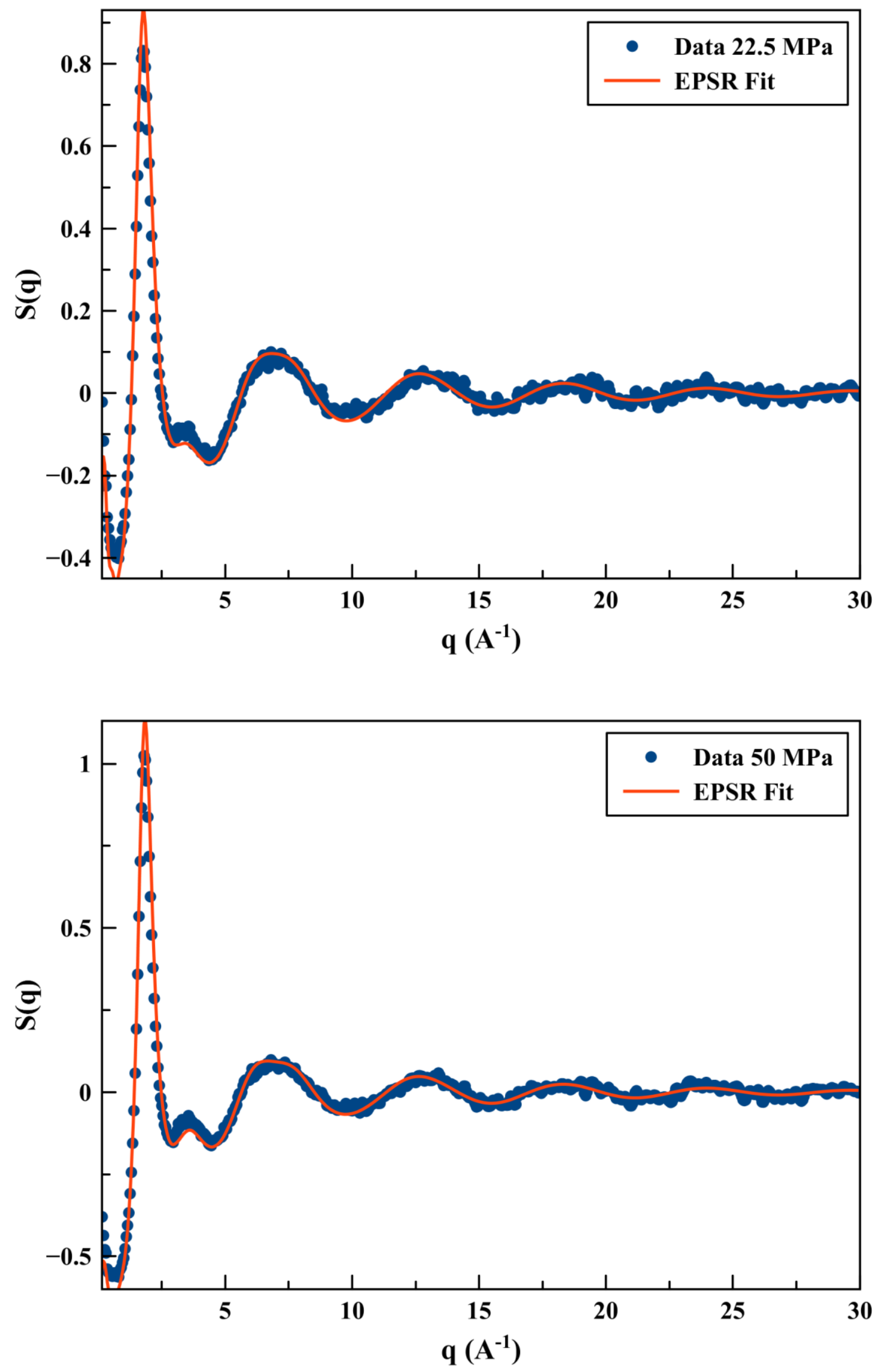

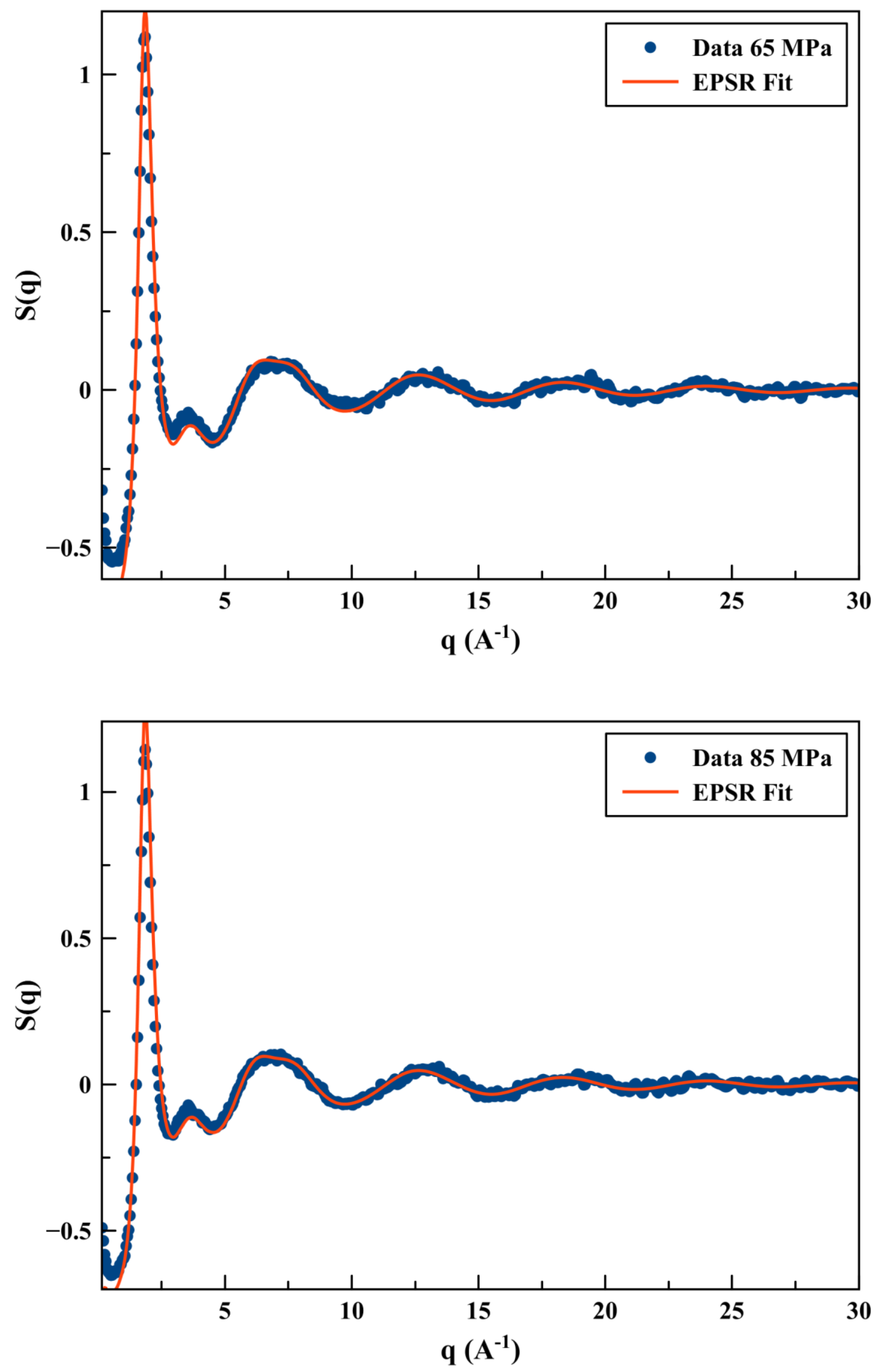

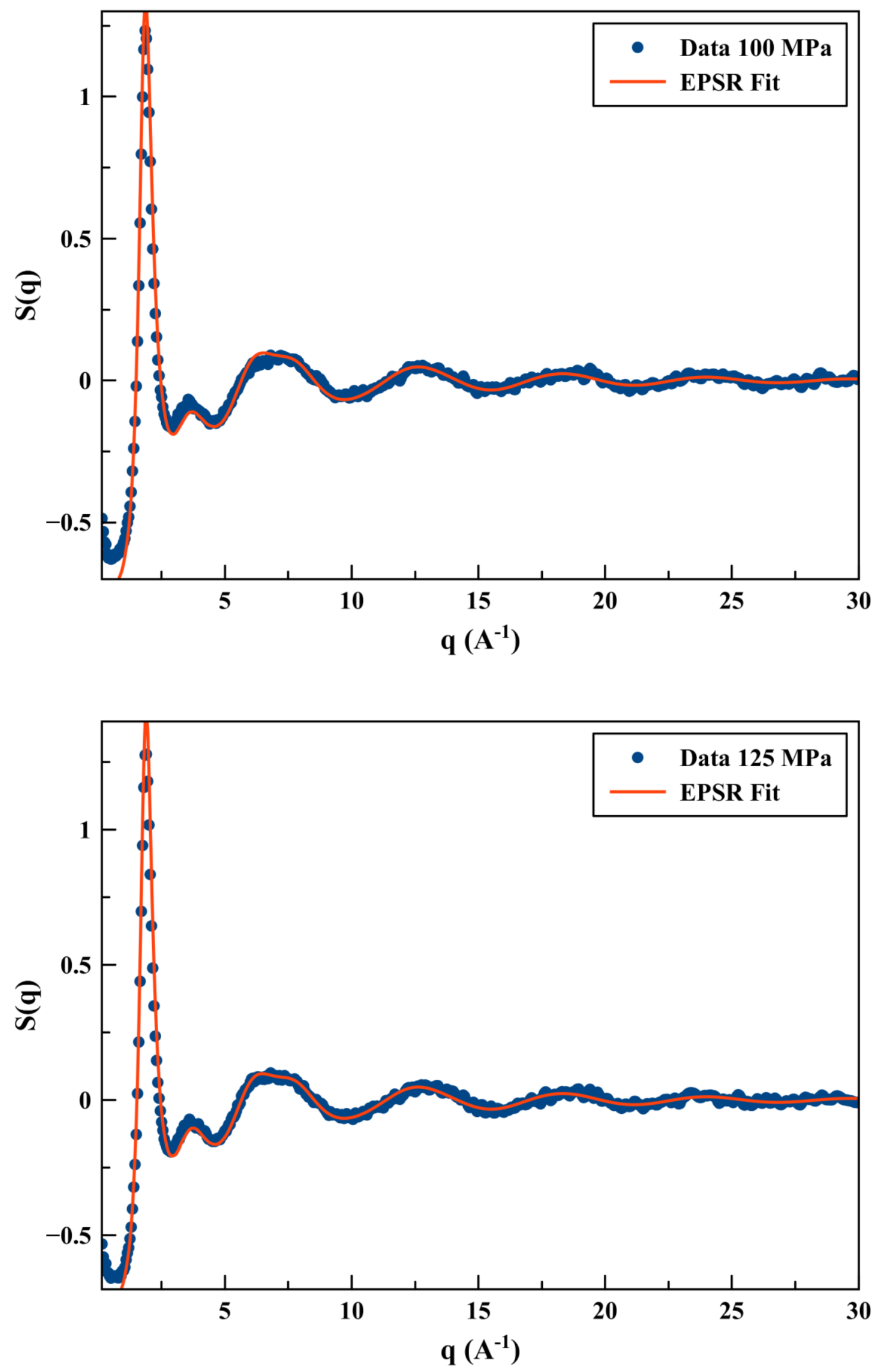


\section{References}

(1) Hannon, A. C. PFIT Correlation Function Fitting Software. http://www.alexhannon.co.uk/ (accessed Jan 18 2011).

(2) Proctor, J. E.; Pruteanu, C. G.; Morrison, I.; Crowe, I. F.; Loveday, J. S., Transition from Gas-like to Liquid-like Behavior in Supercritical N2. J. Phys. Chem. Lett. 2019, 10 (21), 6584-6589.

(3) Soper, A. Tests of the empirical potential structure refinement method and a new method of application to neutron diffraction data on water. Molecular Physics 2001,99, 1503-1516. 